\title{
Improved Model- and View-Dependent Pruning of Large Botanical Scenes
}

\author{
B. Neubert ${ }^{1}$, S. Pirk ${ }^{1}$, O. Deussen ${ }^{1}$ and C. Dachsbacher ${ }^{2}$ \\ ${ }^{1}$ Universitaet Konstanz, Germany \\ ${ }^{2}$ Computer Graphics Group/Karlsruhe Institute of Technology, Germany \\ \{boris.neubert\}@gmail.com
}

\begin{abstract}
We present an optimized pruning algorithm that allows for considerable geometry reduction in large botanical scenes while maintaining high and coherent rendering quality. We improve upon previous techniques by applying model-specific geometry reduction functions and optimized scaling functions. For this we introduce the use of Precision and Recall (PR) as a measure of quality to rendering and show how PR-scores can be used to predict better scaling values. We conducted a user-study letting subjects adjust the scaling value, which shows that the predicted scaling matches the preferred ones. Finally, we extend the originally purely stochastic geometry prioritization for pruning to account for view-optimized geometry selection, which allows to take global scene information, such as occlusion, into consideration. We demonstrate our method for the rendering of scenes with thousands of complex tree models in real-time.
\end{abstract}

Keywords: precision/recall, level of detail, tree rendering

ACM CCS: 1.3 .7 [Computer Graphics]: Three-Dimensional Graphics and Realism- I.3.3 [Computer Graphics]: Picture/Image Generation

\section{Introduction}

Rendering of natural scenes with vegetation as rich as in the real world has been a motivation of computer graphics research ever since. The complex visual appearance and the inhomogeneous structure of botanical objects makes real-time rendering of large scenes a challenging task that extends to this day. The obvious main reason is the tremendous amount of geometry that is needed to represent trees and plants. Storing as well as rendering such objects with full detail is beyond the capabilities even of modern graphics hardware. However, even if processing and rendering the data were possible, then the small sub-pixel details due to the complex geometry can still cause aliasing artefacts.

Many different approaches have been presented to render trees in real-time. Most often simple billboards or impostors [SSK96] are used, or automatically generated billboard clouds [DDSD03, GSSK05] which are sets of billboards that better preserve occlusion and parallax effects. However, these representations are well suited for distant objects and trees, but they are typically over simplified and close views reveal the low quality. It is also not possible to achieve coherent shading of the scene or to adapt the level of detail smoothly and without noticeable artefacts due to the planar nature of billboards [LEST06].

In this paper we present a rendering technique for complex botanical scenes based on pruning. Pruning techniques (stochastically) reduce geometry by simply excluding some parts of the model, for example leaves, from the rendering and correcting contrast and the total rendered area by scaling the remaining leaves [CHPR07]. We improve upon previous methods in several respects:

- We describe a view-optimized pruning instead of purely stochastic simplification of the geometry. This allows us to account for global scene information, for example 
thick and sparse forest and occlusion from neighbouring trees.

- We show that scaling of geometry after pruning should not be inversely proportional to the geometry reduction.

- We formalize this by introducing Precision and Recall as a measure of rendering quality. Our measure does not consider pixel colours, but whether the right pixels of a rendered object are set. We also validate this by conducting a user-study where subjects had to manually adjust the preferred scaling value.

\subsection{Related work}

Since the very beginning of computer graphics, rendering algorithms have been high consumers of computational resources and memory. In general, level of detail (LOD) methods aim at cutting down the rendering cost, mainly by reducing model detail or shading cost. Covering the huge body of work in this field is beyond the scope of this paper. Thus, we refer to Luebke et al.'s excellent textbook [LWC*02] and to the work of Drettakis et al. [DBD*07], which provides an overview over perceptually based rendering and level of detail in this context.

Here we focus on geometry reduction techniques closely related to our work. There exists a variety of texture-based techniques, most notably impostors [SSK96] and billboard clouds [DDSD03], which have been tailored for tree rendering [GSSK05, LEST06]. These techniques generate discrete levels of detail which require special treatment to avoid distracting popping artefacts when adapting the detail, for example using [SW08]. Decaudin and Neyret [DN04] use 3D textures representing parts of a dense forest and aperiodic tiling to render large scenes by volume slicing. However, this method only allows distant views, for example as used in flight simulators. They further extended this approach to volumetric impostors [DN09], but this method shares the drawback of high memory consumption with the other texturebased techniques. Rebollo et al. [RRCR06] describe a GPUfriendly rendering technique for foliage using a multiresolution representation obtained from split and collapse operations, and Gumbau et al. [GCRR11] extend this idea by a view-dependent measure. Both however, require additional memory for storing the multiresolution model.

Because of the aforementioned limitations of these approaches, we base our method on pruning techniques. Especially the simple and efficient idea of stochastically removing geometry makes such approaches extremely simple to use and implement. Stochastic simplification can be easily used with geometry representations that do not require topology information, such as point rendering methods. The QSplat algorithm [RL00] and sequential point trees [DVS03] adapt the size of pre-positioned splats (rendered point primitives) to the required sampling density. Point samples can also be created on the lly, lor example distributed randomly onto surlaces
[WFP ${ }^{\times}() 1$ ], stratified [WS()2] or adaptively [SD()1]. All these methods have in common that - when using fewer samplesthey preserve the total area by scaling the rendered remaining primitives. Klein et al. [KKF* $\mathrm{KK}^{*}$ used a stochastic simplification for polygonal scenes, however, the scene elements are only discarded, not altered, and thus this method is only suitable for coarse previews. Deussen et al. [DCSD02] applied stochastic simplification to rendering complex ecosystems. For reducing geometry they replaced the original triangles successively by lines and then points. Cook et al. [CHPR07] transfer this idea to complex geometry not restricted to point representations. They demonstrate simplification by pruning and scaling adapting not only to an object's screen size, but also to motion blur and depth of field. Their work is closely related to ours, however, we show that the rendering quality can be improved by cleverer scaling. An overview over various further techniques for rendering vegetation, such as fractalbased or space partitioning methods, is given by Zhang and Pang [ZP08].

\section{Improved Scaling for Pruning Algorithms}

In this section we briefly recap the pruning and scaling described by Deussen et al. [DCSD02] and Cook et al. [CHPR07], and discuss the drawbacks of these approaches. Next we introduce the Precision and Recall measure for pruning and scaling, and present the results of our user-study conducted for validation.

\subsection{Area preservation and optimal scaling}

The main objective of simplification algorithms is to preserve the overall appearance of the rendered models while using less geometry and thus reducing rendering cost. Deussen et al. [DCSD02] as well as Cook et al. [CHPR07] propose a simple, and at first sight plausible rule: when the geometry is reduced down to a certain fraction then the remaining geometry is scaled such that the total area of rendered surfaces is equal to the original area. Cook et al. [CHPR07] denote this scaling factor as $s=1 / \lambda$, where $\lambda$ is the fraction of rendered geometry. However, the surface area that is visible after rendering the remaining geometry heavily depends on the actual rendered model. One can easily think of models where a lot of geometry can be removed and they would still cover the same projected area, that is the remaining geometry covers (almost) the same pixels for a certain view direction.

Stochastically pruning the geometry does not only change the area, but also-in particular when pruning stronglythe depth complexity of the rendered model. Cook et al. [CHPR07] account for this by calculating the expected visible area of a subset of randomly chosen elements of a model, and adapt the scaling factor accordingly. Our results demonstrate an important and interesting fact: the largest decrease in rendering quality due to wrong scaling does not occur for strong, but for slight and moderate pruning, where the 
Table 1: Classification of pixels for Precision and Recall.

\begin{tabular}{ll}
\hline Set & \multicolumn{1}{c}{ Pixels that are... } \\
\hline true positives & $\begin{array}{l}\text {...correctly set, i.e. rendered for the original and } \\
\text { for the simplified model. }\end{array}$ \\
false positives & $\begin{array}{l}\text {.. wrongly set, i.e. rendered only for the } \\
\text { simplified model. }\end{array}$ \\
false negatives & $\begin{array}{l}\text {.. rendered for the original model, but not covered } \\
\text { by the simplificd onc. }\end{array}$ \\
\hline
\end{tabular}

depth complexity correction has only little influence. In the next section, we introduce the Precision and Recall measure which does not only take the number of pixels but also their classification into correctly set and unset pixels into account.

\subsection{Precision and recall}

Precision and Recall (PR) are well-known statistical classifications or measures for exactness and completeness. They are widely applied in the domain of information retrieval and are closely related to sensitivity and specifity to measure the performance of binary classification algorithms, such as support vector machines and Bayesian networks [Rij79].

Precision is defined as the ratio of correctly identified items (true positives) to both correctly and incorrectly identified items (sum of true positives and false positives). In our case, when rendering a pruned model it is the ratio of pixels that are correctly set, that is they would have been rendered for the full-detail model as well, and the total number of set pixels.

Recall is the quotient of correctly identified items (true positives) and all relevant items (sum of true positives and false negatives). Again translated into this scenario: the ratio of correctly set pixels and the number of correctly set pixels plus the number of pixels that should have been rendered, but which are not covered by the pruned model.

Thus, PR are defined as (true positives $t p$, false positives $f p$ and false negatives $f n$ ):

$$
P=\frac{t p}{t p+f p} \quad \text { and } \quad R=\frac{t p}{t p+f n} .
$$

Table 1 gives an overview of the relevant pixels sets, which are shown in Figure 1 for a simple example. More formally, we denote the set of all pixels covered by the original model as $P_{\text {orig }}$, and the set of pixels rendered for the simplified model as $P_{\text {simplified }}$ and thus get:

$$
\begin{aligned}
& t p=\left\{p \mid\left(p \in P_{\text {simplificd }}\right) \wedge\left(p \in P_{\text {orig }}\right)\right\} \\
& f p=\left\{p \mid\left(p \in P_{\text {simplificd }}\right) \wedge\left(p \notin P_{\text {orig }}\right)\right\} \\
& f n=\left\{p \mid\left(p \notin P_{\text {simpliticd }}\right) \wedge\left(p \in P_{\text {orig }}\right)\right\} .
\end{aligned}
$$

Precision and Recall are reflecting how well the simplified model is representing the information-in our case the
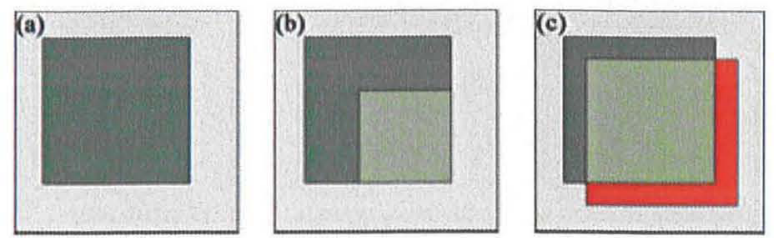

Figure 1: (a) Dark green: pixels covered by original 'model'. (b) Light green: pixels covered by the 'model' rendered with reduced geometry and without scaling: no additional pixels are covered and thus only the Recall value is affected, whereas the Precision score remains 1. (c) The simplified and scaled 'model' covers pixels that were not covered by the original model (red,false positives). Dark green pixels are false negatives, light green ones are true positives.

rendered pixels - compared to the original model. The PRscores for the original model, that is rendering at full detail, are $P=1.0$ and $R=1.0$. The big advantage of $\mathrm{PR}$ is that not only the number of pixels is taken into account, as the preservation of the projected area does, but PR is also sensitive to whether the same pixels are covered. Thus, models rendered with reduced geometry that cover almost the same pixels as the original model will get PR-scores closer to the optimal $P=1.0$ and $R=1.0$.

Using PR-scores, we can now define the optimal scaling value, $s_{\text {opt }}$, depending on the fraction of rendered geometry, denoted as $\lambda$ (similar to Cook et al. [CHPR07]). The underlying idea of our heuristic is that one unset pixel that should have been covered is as bad as a set pixel that should not have been covered. Consequently, we choose $s$ in a way such that we minimize the distance of the point $(P(s), R(s))$ (in the PR diagram) to the optimal PR-score at $P=1$ and $R=1$ :

$$
s_{\mathrm{opt}}(\lambda)=\underset{s}{\operatorname{argmin}} \sqrt{(1-P(s, \lambda))^{2}+(1-R(s, \lambda))^{2}} .
$$

Figure 2 shows PR results for different models, values of $\lambda$, and scaling values $s$. To determine the optimal scaling, $s_{\mathrm{opt}}$, for a given tree model we equidistantly sample $\lambda$ in a preprocessing step and compute the respective PR-scores. During rendering, we linearly interpolate $s_{o p t}$ for non-tabulated $\lambda$-values. An interesting, but also important, property of our PR measure is that graphs for increasing $\lambda$ are ordered towards the upper right corner. This reflects the intuitive assumption that using more geometry better resembles the original model.

\subsection{Experimental validation and user study}

We carried out our user study with 19 subjects (both experienced and unexperienced in computer graphics) presenting an unpruned, full detail model side-by-side with a simplified version of the same model. The subjects were asked to choose 

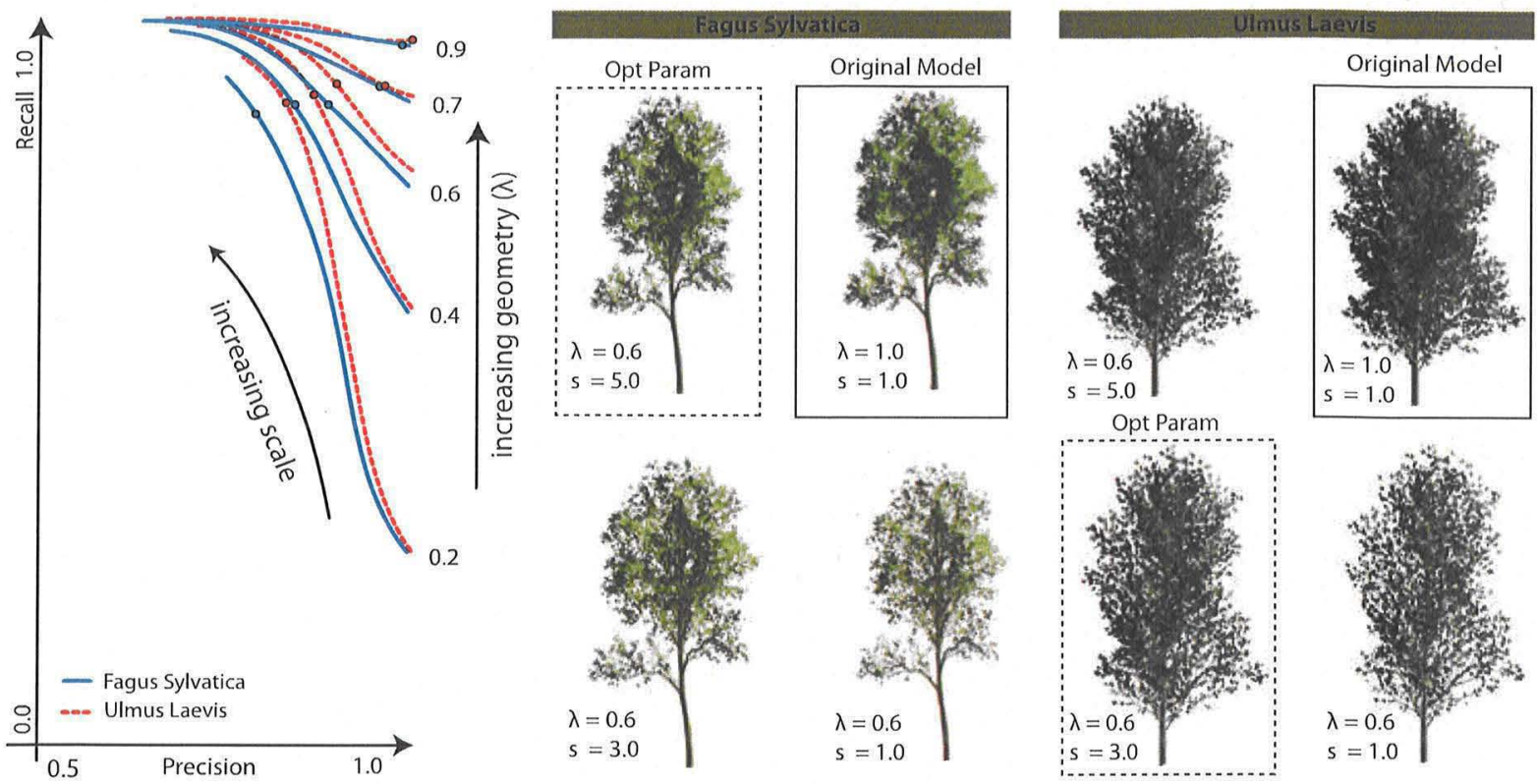

Figure 2: The Precision-Recall diagram for different plant models, five different geometry levels $\lambda$, and varying scaling values. An interesting case is the Ulmus model (right): it does not benefit fiom scaling for higher $\lambda$-values, and scaling even lowers the PR-score, that is the distance of the PR-coordinate to the top-right corner which represents the optimal score of $P=1$ and $R=1$.

the scaling value, for a given $\lambda$, such that the appearance of the reduced model resembles the full-detail model as close as possible (Figures 3 and 4). This procedure has been done for 10 geometry levels and five different tree models.

The study revealed that in particular for little simplification $(\lambda>0.8)$ the user-preferred scaling values were not only considerably different for every model, but also on average smaller than the scaling values computed according to Cook et al. [CHPR07]For smaller values of $\lambda$ the standard deviation of the preferred scaling increased considerably, however, the
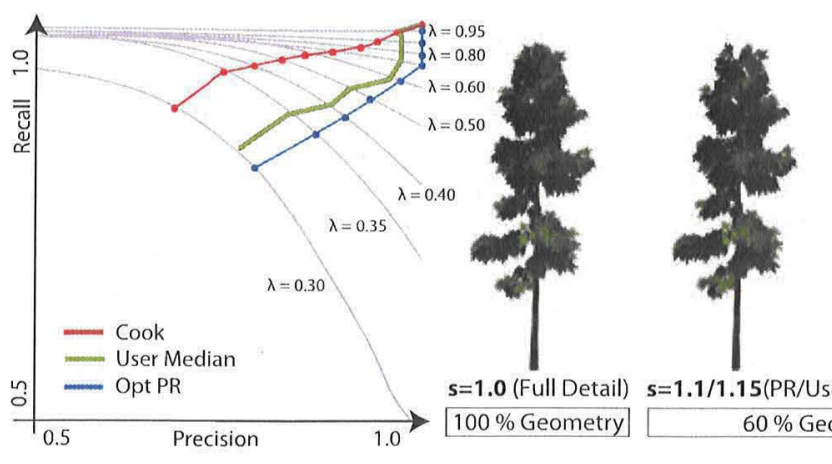

$\mathbf{s = 1 . 0}$ (Full Detail)

$=\mathbf{1 . 1} / \mathbf{1 . 1 5}$ (PR/User) s=1.65 (Cook) $60 \%$ Geometry median value was typically very close to our $s_{\text {opt }}$. The large standard deviation can be explained by the fact that these geometry levels are actually only used to render trees at large distances, whereas the model presented in the user study was rendered at full size. This obviously makes it harder to judge the appearance of the model and led to larger deviations in the preferred scale value.

Figures 3 and 5 show the results of the user study. The preferred scaling values are shown in the respective PR diagram in green, next to $s_{\mathrm{Cook}}$ and $s_{\mathrm{opt}}$. On the left in Figure 5 the
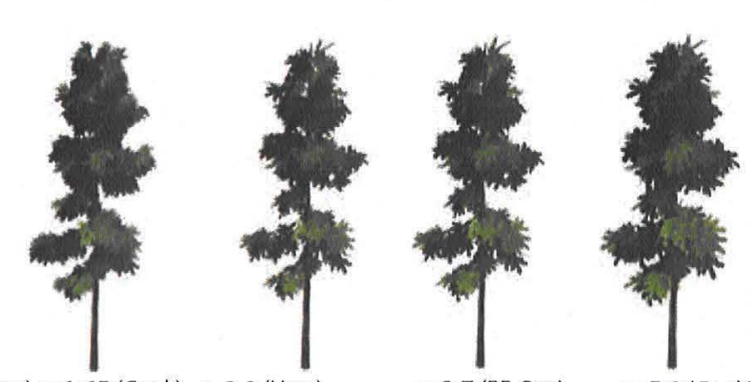

$\mathbf{s}=\mathbf{2 . 7}$ (PR Opt)

$s=5.0($ Cook $)$

Figure 3: Comparison between different scale values for Picea Abies. Red: scale value according to Cook et al. [CHPR07] $s_{\text {Cook }}=1 / \lambda$. Green: user-preferred scale value (median). Blue: optimal scaling value found using PR-scores. Scaling does not improve the PR-scores for this model when more than $60 \%$ of the original geometry is rendered. The reason is that this model exhibits very dense geometry, and pruning does not immediately intpact the overall appearance. This is also reflected in the preferred scaling values obtained from the user study. 


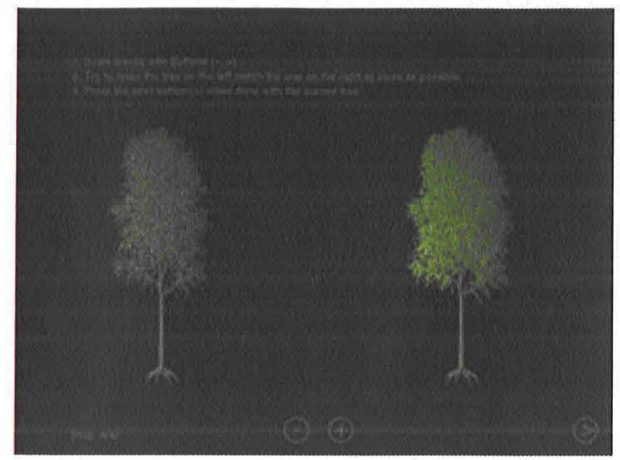

Figure 4: This screenshot shows our implementation for the user study: the subject is asked to adjust the scaling factor (for different $\lambda$-values) for the left model, such that the rendering matches the full detail rendering on the right as close as possible.

values are shown in a PR diagram and additionally the scaling values with respect to the different $\lambda$ values on the right. For two models the user selected values are in general larger than $s_{\text {Cook }}$, for the Picea Abies model the user selected values are smaller; in all five cases our method faithfully predicts suitable scaling values.

\subsection{Impact of scaling and view direction}

Precision and Recall are defined in image space and therefore view-dependent measures. However, our experiments indicate that for natural objects, which we target in our work, the deviations in the measure are very small. This is because
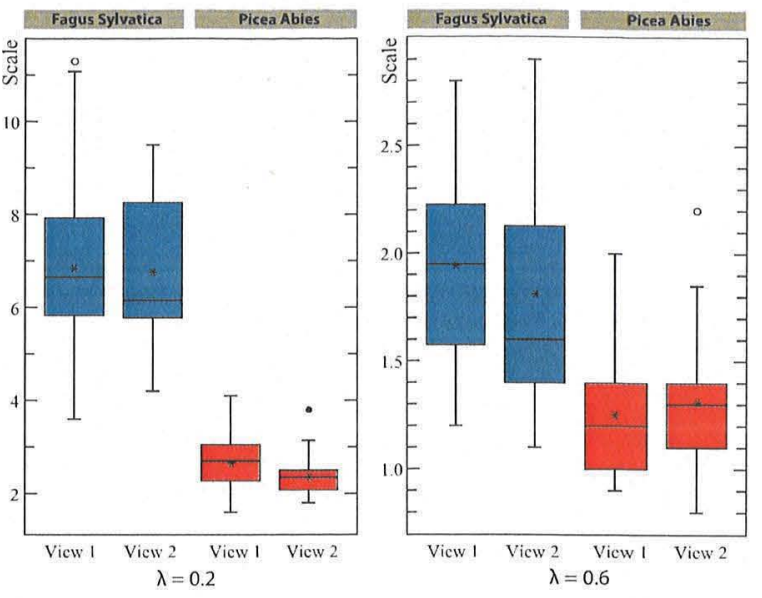

Figure 6: Comparison of the user selected scaling values for two different tree models and two geometry levels (left: $\lambda=$ 0.2 , right: $\lambda=0.6$ ). For the Fagus Sylvatica model the users preferred larger scaling values (independent of the viewing direction) compared to the Picea Abies model. Besides the view independence the large difference in preferred scaling values for different plant models is significant and underlines the need for model dependent scaling. Outliers are indicated as circles, and the user average as stars.

such objects, for example trees, typically do not have a dominant view direction but rather uniformly distributed normals and vertex positions. This was also confirmed by our user study where we analysed user-selected scaling values for two different views of every model and geometry level (Figure 6). For both views, the variance and mean are very close

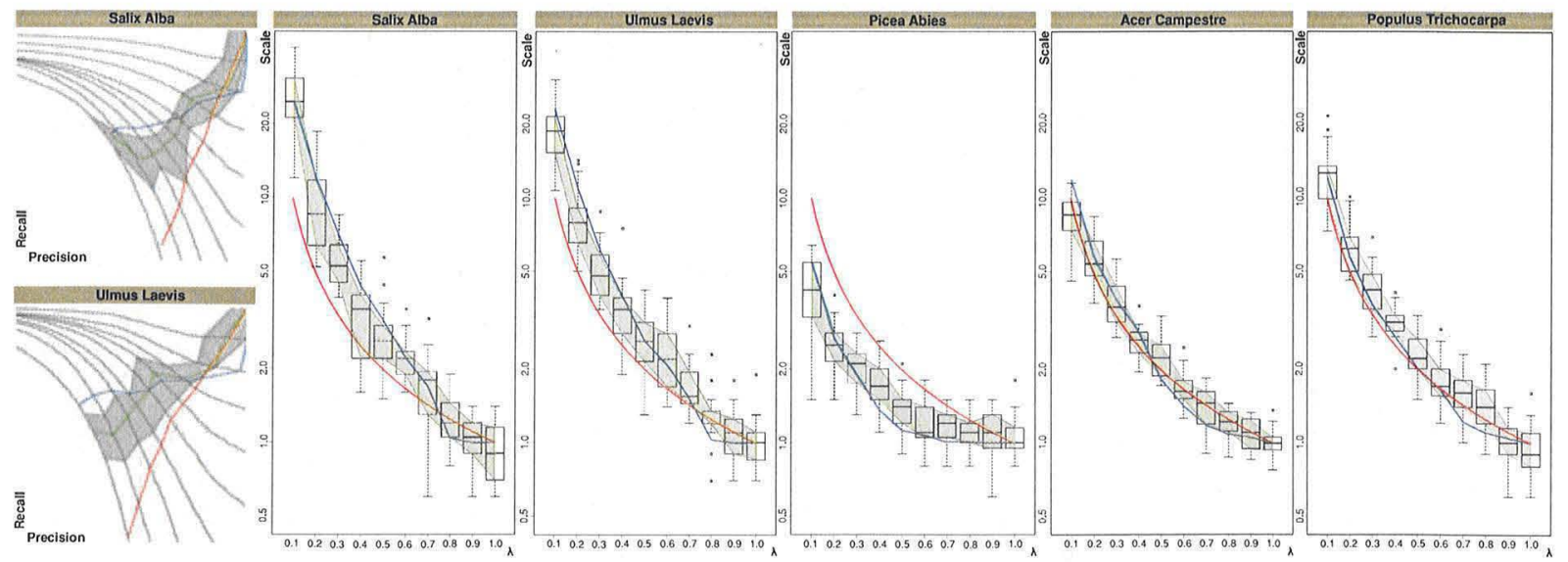

Figure 5: Comparison between user preferred scaling values indicated in green (user median, first and third quartile), scaling according to cook (red) and scaling predicted by our method (blue). The $\lambda$-values of the PR-diagrams (left) are sampled with step size 0.1 starting at $\lambda=0.1$. The diagrams on the right show the user selected scaling values for the same $\lambda$-levels as on the left. Although the user preferred scaling values for the Picea Abies model are significantly lower than the suggested Cook scaling, the scaling values for Salix Alha and Ulmus Laevis are higher. In all five cases, the optimal scaling values predicted with our method are in close range to the user preferred values. 
(Fagus Sylvatica: $s\left(\lambda=0.2, V_{1}\right)=6.89$ and $s\left(\lambda=0.2, V_{2}\right)=$ $6.75 ; s\left(\lambda=0.6, V_{1}\right)=1.94$ and $s\left(\lambda=0.6, V_{2}\right)=1.81$. The variance analysis (ANOVA) of the user-study data indicates that the hypothesis ( $H_{0}$ : mean is the same for both views) can be accepted with $F_{V 1}=0.07$ and $F_{V 2}=0.62$ for Fagus Sylvatica, and $F_{V 1}=2.66$ and $F_{V 2}=0.14$ for Picea Abies, well below the critical F-Value of $F_{\text {crit }}(1,36)=4.11$ for $\alpha=$ 0.05 .

A very important property of the PR measure, and thus for the application of our method, is that PR scores are invariant to rendering the models at different screen sizes, that is scaling the model without changing $\lambda$. This can be explained as follows: when reducing the size of a (pruned) model it is more likely that multiple triangles are projected onto the same pixels, and thus it is also more likely that all pixels covered by the original model and also covered by the pruned one.

\subsection{Detail level selection}

Rendering complex scenes is only possible if we reduce the level of detail for distant trees and only render with high quality when trees are close to the camera. Using the PRscore from Section 2.2, we define the quality $Q$ of a rendering as the distance of the PR-vector to the optimal value $(1,1)$ :

$$
Q(\lambda, s)=1-\sqrt{(1-P(s, \lambda))^{2}+(1-R(s, \lambda))^{2}} .
$$

For rendering we want to ensure that a tree at a certain distance, $d$, to the camera will be rendered at a given minimum quality. This means that the PR-vector for a model rendered with a geometry level $\lambda(d)$ has to be within a certain proximity to the top-right corner of the PR-diagram (Figure 7). That is, for rendering we need to sample and store the function $\lambda(d)$ to provide this desired minimum quality for a given $d$. Note that determining this function takes place in a precomputation step for every tree model.

There are various options to define minimum quality, for example letting the user define a given maximum deviation from the optimal PR-scores and computing $\lambda(d)$ accordingly. To compare our method to Cook et al.'s, however, we determine $\lambda(d)$ such that the rendering quality of our PRoptimized pruning and scaling matches their quality for the same distance $d$. That is, we render a model with the same PR-scores, but with less geometry if possible.

This works as follows: Cook et al. use $\lambda_{\text {Cook }}(d)=(1-d)^{2}$ (with $d$ normalized to $[0 ; 1]$ ) as a simple relation of distance and geometry. Rendering the model with this pruning yields a quality $Q\left(\lambda, s_{\text {Cook }}\right)$. Next, we determine the smallest $\lambda_{\text {opt }}$ whose rendering with the optimal scaling $s_{\text {opt }}\left(\lambda_{\text {opt }}\right)$ (Section $2.2)$ yields equal or better quality, that is $Q\left(\lambda_{\text {opt }}, s_{\text {opt }}\right) \geq$ $Q\left(\lambda, s_{\text {Cook }}\right)$. This compound mapping yields a $\lambda_{\text {opt }}$ and an associated $s_{\text {opt }}$ for a given view distance $d$. Obviously this pre-computation can only be carried out for a finite number of values. Therefore, we use 10 equidistant samples in $[0 ; 0.1)$
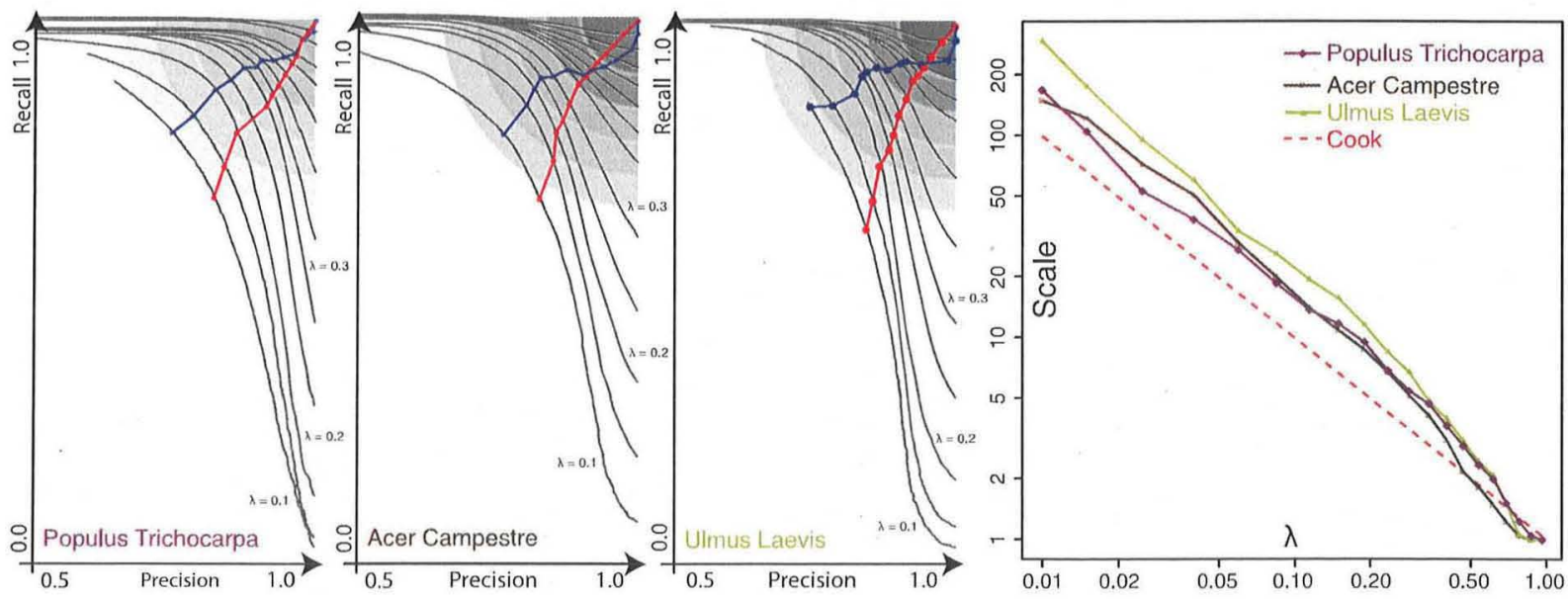

Figure 7: The first three graphs show PR-values for three different tree models. The blue graph shows the optimal scaling values $s_{\mathrm{opt}}$, the red one the standard scaling values $s_{\mathrm{Cook}}$ (according to Cook et al.). For rendering we choose $\lambda$ such that we maintain a minimum quality that is required for a given viewing distance. The minimum quality requirements are indicated by the circles centred at the top-right corner of the PR diagram. As we can see, it is possible to reduce the geometry to a larger extend for model Populus Trichocarpa compared to model Acer Campestre, in particular for small values of $\lambda$. Right: a log-log plot of scale versus fraction of remaining geometry, that is $\lambda$. Note that the connected $\left(s_{\mathrm{opt}}, \lambda\right)$ and $\left(s_{\mathrm{Cook}}, \lambda\right)$ lines in the PR diagram (left three plots) look very rough. The values plotted against the geometry level $\lambda$, however, are smooth with the expected exponential behavior: The smoothness is important to avoid popping artefacts during rendering. The $\lambda$-values are sampled with 0.05 step size from $\lambda=0.1$ to 0.4 and with step size 0.1 above. 
and $[0.1 ; 1.0)$, respectively, and linearly interpolate $\lambda_{\text {opt }}$ from the stored samples.

Figure 7 shows the mapping of distance to pruning for three different tree models. The plots show that model (a) and (c) can be rendered with high quality (PR-score within the second circle depicted in Figure 7) even for a low value of $\lambda=0.2$. For model (b) a higher $\lambda$-value is required even for larger viewing distances. The plots of rendering with higher $\lambda$-values reveal that model (b) and (c) suffer stronger from pruning, while model (a) preserves most of the rendered pixels of the original model.

\section{Rendering Priority}

The rendering priority reflects the order in which geometry is removed from the original model with decreasing $\lambda$. Cook et al. [CHPR07] tried to avoid correlation in the rendering priority between order and position, size, surface normal and colour as much as possible, and thus prevent disturbing artefacts when rendering with reduced geometry. However, they stated that in some cases the priority order might be found procedurally. In this section we will propose different ways to find the rendering priority order algorithmically in a way that ensures higher Precision and Recall values. Note that it is only the PR-scores that make it possible to compare different prioritization heuristics.

\subsection{Silhouette preservation and density normalization}

To optimize the rendering priority we need to determine which parts of a model are close to the boundary and will potentially be part of the silhouette, and which regions exhibit a high or low density of geometry.

To this end, we will need to define what the 'boundary' of a (botanic) model is. For this, we use implicit surfaces that tightly enclose a model. They have also been used to generate normal distributions for such models that provide more realistic and expressive illumination of foliage [LBD07]. Implicit surface can be generated using metaballs [Bli82]: first, a set of generation

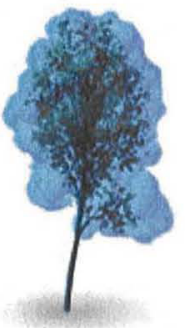
points $P$ is chosen and an influence radius $r_{i}$ is assigned to every point. As generation points we use the centre of leaf-triangles and choose influence radius proportional to the overall plant height ( $5 \%$ in our case). The contribution of a single generator point $p_{i} \in P$ at a point in space, $q$, to the global density function is defined as:

$$
D_{i}(q)=\left(1-\left\|q-p_{i}\right\|^{2} / r_{i}^{2}\right)^{2} .
$$

The sum over the contributions of all $p_{i}$ yields the global density function: $F(q)=\sum_{i} D_{i}(q)$. An iso-surface is then defined by a given iso-value $a$ with $F(q)=a$, and can be

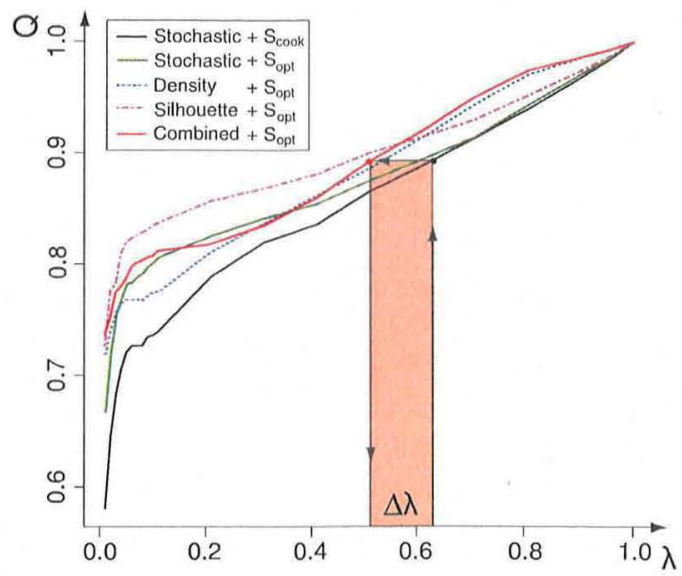

Figure 8: Comparison of prioritization heuristics to pure stochastic ordering for the Ulmus model. The red area shows the benefit of switching fiom stochastic order with $s_{\mathrm{Cook}}$ to the combined prioritization with $s_{\mathrm{opt}}$. It is possible to maintain the quality with less geometric detail, denoted as $\Delta \lambda$.

triangulated using marching cubes (see inset). To extract a tree's tight hull we choose an iso-value such that $a=0$.

In the following we will discuss different prioritization heuristics based on the global density function. Again, we measure the quality using PR-scores.

\subsubsection{Varying density}

First, we use the global density function to identify regions of high geometric density within a model. Triangles that are close to each other are likely to be projected to the same location in image space. Thus, removing triangles in very dense region lowers the probability of overdraw while still keeping chances high that all original pixels are covered even without scaling the remaining geometry. We evaluated this guided geometry prioritization using our PR measure, and experiments showed that the quality improves for high values of $\lambda$. For such values, there are larger variations in local density (Figure 8), as no, or little, density controlled pruning did take place. These variations obviously vanish when reducing more and more geometry prioritized in dense regions, which makes the density variation become more uniform. When this point is reached, that is for smaller $\lambda$, we switch back to pure stochastic prioritization. The performance increase due to density prioritized pruning depends on the variance of $F(q)$ within a model, and thus models with almost uniform density do not benefit from this strategy.

\subsection{Orientation}

As second heuristic we investigated the improvement of rendering prioritization based on the deviation between a 
triangle's normal and the normal on the nearest point on the implicit surface (denoted as $\alpha$ ). Preserving geometry facing outwards, that is small $\alpha$, generally enforces a pixel coverage of the rendered model that is closer to that of the full detail model. Scaling triangles that resemble the models iso-surface turned out to perform well, especially for small values of $\lambda$ (Figure 8).

Pruning triangles close to the implicit surface with lower probability, however, leads to inferior results. Although at first sight it seems reasonable to preserve the silhouette, keeping and scaling triangles close to the surface results in many false positive pixels, in particular for strong scaling with small $\lambda$. This leads to visible artefacts and low Precision and Recall scores.

\subsection{Combined prioritization}

Both heuristics determine 'survival probabilities' for the triangles of a model. In our implementation we use an empirically found weighting to combine both of them, where the orientation heuristic has smaller impact. We choose $P_{\text {Combined }}=\left(P_{\text {Silhouette }}\right)^{2}+P_{\text {Density }}$ normalized to $[0,1]$ and then sort the triangles for descending survival-probability (adding a small amount of randomness) to obtain a single list representing the entire model. Similar to Sequential Point Trees [DVS03], we can then render only a prefix of the list, according to $\lambda$, to render a pruned model.

\section{View-Dependent Optimization}

Considering the viewing distance and thus the projected size of a model is of course one important aspect for choosing the geometry level. However, occlusion also has impact on the required detail: partially occluded trees, or trees that are completely surrounded by others, do not contribute considerably to the scene's appearance and thus should be rendered using less geometry. In this section we show how we can determine occlusion of trees at run-time, and control our rendering accordingly.

For this we analyse the occlusion of each tree by testing the visibility of a set of sample points distributed on its iso-surface. In principle, there are various possibilities to perform the visibility test, for example ray casting, or using some form of pre-computed visibility information. To facilitate real-time rendering without pre-computation, we use an image space approach relying on the depth buffer of the camera image only. Obviously, this depth buffer is not available before actually rendering the geometry. However, if we assume smooth camera movement we can exploit frameto-frame coherency and test the visibility of sample points using the depth buffer and transformation of the previous frame. For abrupt movements, or sample points that are projected outside the viewport, we conservatively assume full

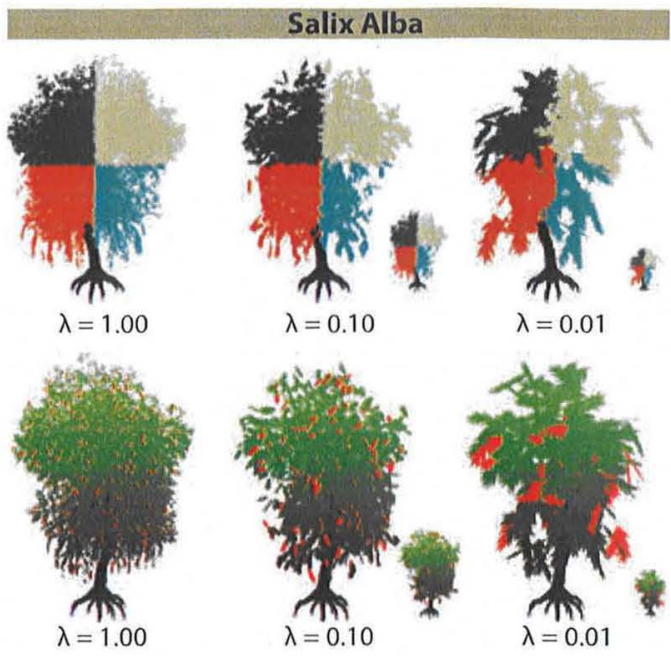

Figure 9: Effect of model simplification to intra-model colour variation: boundaries of large coloured regions are maintained even for low detailed models (top row). Smooth gradations do not exhibit noticeable changes under stochastic pruning, apart from effects similar to colour quantization. The arrangement of small details (e.g. the red leaves) obviously changes, but still does not cause flickering. Note that strong pruning typically occurs for distance trees.

visibility and thus render the models at possibly higher detail than actually necessary.

From the visibility of the sample points we then deduce an approximate occlusion factor for each tree. The fraction $f$ of visible to the total number of sample points is used to control $\lambda$, in a way such that $\lambda_{\text {occl }}=\lambda_{\text {opt }}(d) \cdot \max (f, 0.1)$. To avoid popping artefacts, we use a hysteresis function and smooth the $\lambda_{\text {occl-values over time. }}$

\section{Colour Variation}

Our method is suitable for rendering (groups of) objects that are aggregated from a large number of randomly oriented and placed geometric details. Typically we can also assume a near uniform colour distribution, or large-scale gradations, for botanical objects. Cook et al. [CHPR07] intentionally do not correlate the rendering priority order to the colour distribution or any other model characteristics. Apart from colour variation Cook et al. proposed a method to preserve colour contrast during simplification. In this section, we show the effect of our pruning algorithm on three different kinds of colour variations (Figure 9). Although large coloured regions across the objects are preserved (Figure 9, top), smooth colour gradations show the effects similar to quantization artefacts, which is expected due to the geometry reduction 


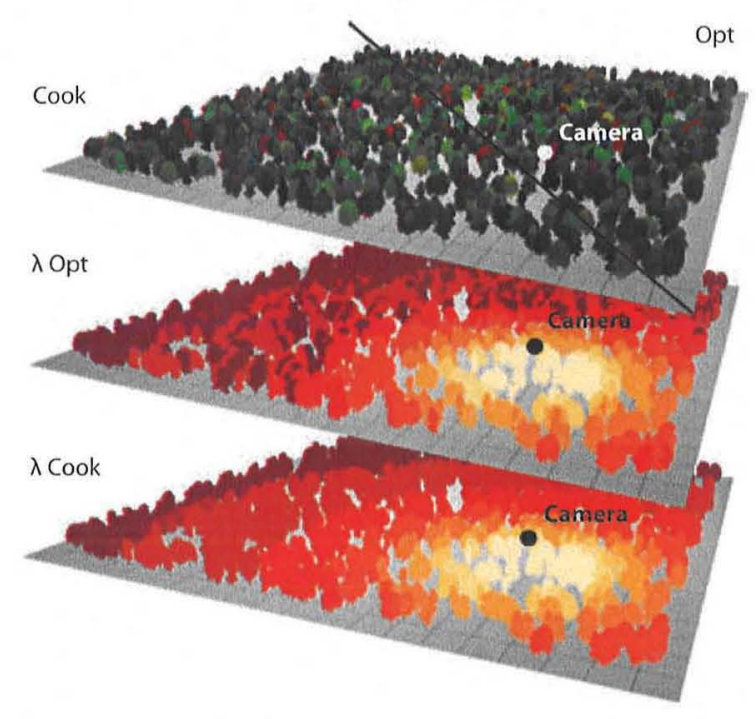

Figure 10: A scene with intentionally exaggerated high inter-model colour variance. Even for distant areas with a low amount of geometry individual tree models can be identified (top layer). Bottom two layers: geometry distribution according to Opt and Cook.

(bottom). The pruning becomes most apparent for small, randomly distributed and salient details (red leaves in Figure 9). Under strong pruning the fraction of covered pixels is still preserved, but the distribution becomes less random due to the smaller number of samples (bottom right). Note that all these artefacts become less apparent if the model is rendered with a size according to the geometry level. Colour variations between models are of course preserved, as we do not prune across objects, and thus individual trees can still be identified (Figure 10).

\section{Results and Comparison}

In this section we present results of our method and comparisons to Cook et al.'s [CHPR07] method to assess rendering performance, and to billboard clouds [DDSD03] to demonstrate the benefits of our (view-dependent) pruning over texture-based representations. We implemented our method using OpenGL and performed all tests and measurements using an Intel Core i7 at $2.8 \mathrm{GHz}$, with $4 \mathrm{~GB}$ of memory, and a NVIDIA Geforce GTX 295 GPU.

\subsection{Comparison to billboard clouds}

For rendering botanical models, most real-time applications resort to a representation with relatively few textured polygons recreating the original model. For video games these models are often created manually, although billboard clouds [DDSD03] can be used to obtain such reduced models

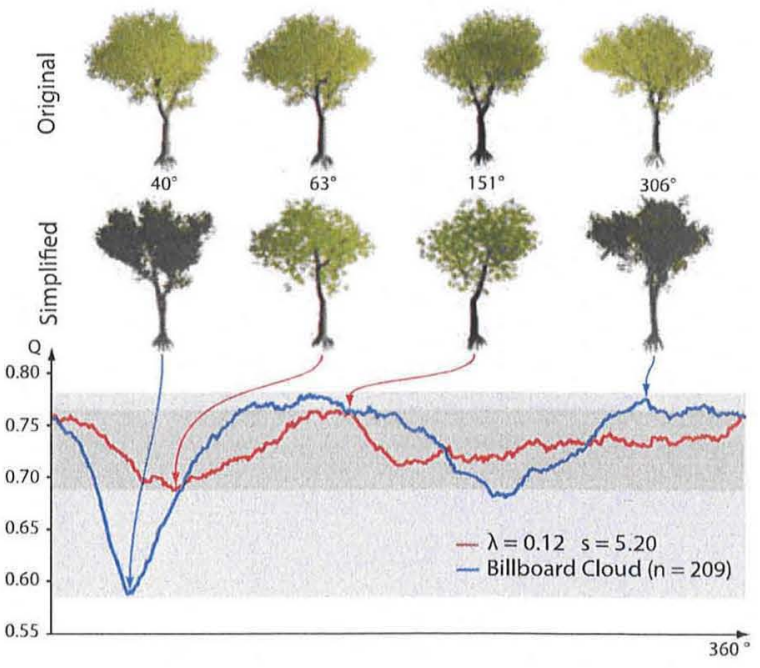

Figure 11: Comparison of rendering a 360 rotation of a billboard cloud model (209 billlboards) and a pruned model $(\lambda=0.12, s=5.2)$. The parameters for the latter are chosen to match the average quality $Q$ of the billboard model. Note that the variance for the billboard model is much higher:

automatically. Note that these representations do not provide a 'continuous' level of detail and switching between different levels is prone to popping artefacts. We compared our results to billboard clouds by evaluating the rendering quality according to our measure $Q$ (Section 2.5). We observed that the rendering quality varies strongly with the view direction when using billboard clouds, and significantly less with our optimized pruning. Figure 11 shows this comparison where the parameters of our pruning are adjusted to match the average quality of a billboard representation. Note that another applications of our PR-measure can be the billboard cloud generation itself, where it can be used to identify bad views for which the billboard representation needs to be improved.

\subsection{Rendering performance}

Our method allows us to render complex scenes with 5000 tree models at interactive to real-time rates, that is $8-25$ frames per second at a resolution of $1600 \times 1200$ (Figure 12). The full-detail geometry of the scene consists of more than 1.3 billion vertices and renders at only 0.8 frames per second on the same hardware, that is far from interactive speed. Our optimized and prioritized pruning, together with the view-dependent visibility tests reduces the number of vertices per frame to about 26 million vertices. On average this yields a performance increase, compared to Cook et al., of approximately $60-70 \%$ while maintaining the same quality (determined using the PR scores). Figure 10 shows a complex scene with exaggerated colour variation, individual tree models can still be identified even for low geometry 


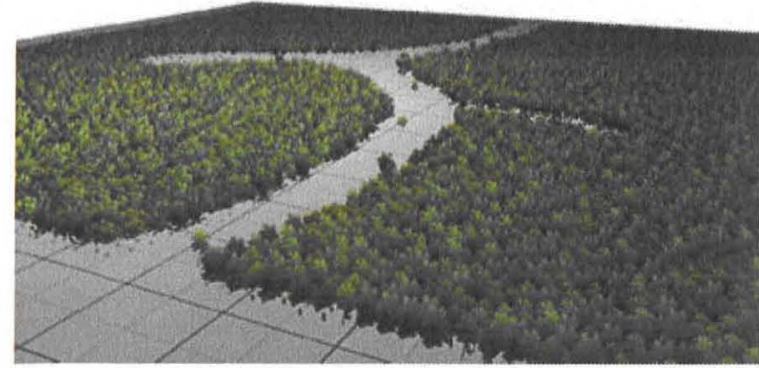

Figure 12: This scene consists of 5000 trees (in total 1.3 billion vertices). With our optimized pruning we can render this scene with 15 frames per second when all 5000 trees are visible. Pruning according to Cook et al. [CHPR07] renders an image of the same quality at 9 frames per second.

levels (top layer). The bottom two layers visualize the colourcoded geometry level. Although $\lambda_{\text {Cook }}$ is chosen depending on the camera distance $\lambda_{\text {Opt }}$ is individually chosen for each tree model according to Section 2.5. Figure 13 shows a detailed evaluation of a standard camera path through a scene with 1276 trees without culling following the equal quality approach. Although choosing an optimal scaling value with stochastic prioritization $\left(S_{\text {opt }}\right.$, Figure 13 green graph and Table 2) already gives on average a $10 \%$ geometry reduction (choosing a lower geometry level with equal PRscores), additionally changing the prioritization according to the proposed heuristics gives an average geometry reduction between $17 \%$ and $40 \%$ (Table 2). Although the combined prioritization performs better for close views (Figure 8) for

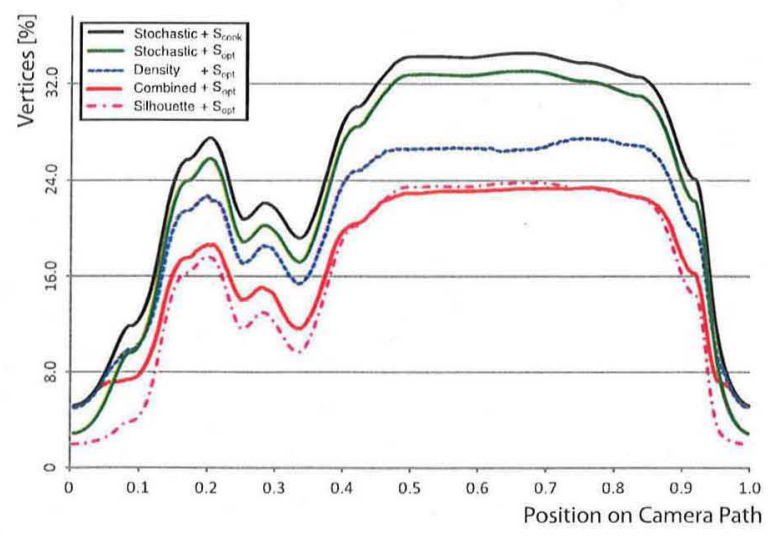

Figure 13: Performance evaluation of different heuristics for a camera path through a scene with 1276 tree models without culling ( $\sim 63 \mathrm{M}$ vertices). It can be seen that the silhouette based prioritization performs best for distant views (start and end of the camera path). Although the combined prioritization performs slightly better in walk through camera positions.
Table 2: Geometry reduction with our different prioritizations (Figure 13) compared to Cook et al. [CHPRO7]. For example, the combined heuristic requires $30.1 \%$ less geometry on average to render at the same quality as Cook et al.

\begin{tabular}{lcccc}
\hline & $\begin{array}{c}\text { Stochastic } \\
+S_{\text {opt }}\end{array}$ & $\begin{array}{c}\text { Density } \\
+S_{\text {opt }}\end{array}$ & $\begin{array}{c}\text { Silhouette } \\
+S_{\text {opt }}\end{array}$ & $\begin{array}{c}\text { Combined } \\
+S_{\text {opt }}\end{array}$ \\
\hline Avg & 0.101 & 0.174 & 0.407 & 0.301 \\
Min & 0.043 & 0.022 & 0.308 & 0.014 \\
Max & 0.437 & 0.236 & 0.693 & 0.392 \\
\hline
\end{tabular}

most camera positions in the test scene with a mixture of distant and close models the silhouette-based prioritization performs best.

\section{Conclusions and Future Work}

In this paper, we introduced Precision and Recall as a measure of quality for rendering complex geometry with pruning. We further improved on previous methods by applying model-specific geometry reduction and optimized scaling as well as view-optimized pruning. We evaluated our method by means of a user study that indicates a considerable improvement compared to naive and purely stochastic pruning. However, our work also raises new questions. One interesting direction of future research is to consider more than just correct and incorrect pixels in PR, for example by accounting for deviations in the normals, measuring contrast and colour differences, or to evaluate how visible differences predictors can improve the measure and whether their use amortizes.

\section{Acknowledgments}

The authors thank the reviewers for their insightful comments. This work is supported by DFG Research Training Group GK-1042 Explorative Analysis and Visualization of Large Information Spaces, University of Konstanz and the project Information at your Fingertips Interactive Visualization for Gigapixel Displays, which is supported by the 'Information Technology Baden-Württemberg (BW-FIT)' funding program.

\section{References}

[Bli82] Blinn J. F.: A generalization of algebraic surface drawing. ACM Transaction on Graphics 1, 3 (1982), 235-256.

[CHPR07] Cook R. L., Halstead J., Planck M., Ryu D.: Stochastic simplification of aggregate detail. ACM Transactions on Graphics (Proceedings of SIGGRAPH 2007), 26, 3 (2007), article no. 79. 
[DBD*07] Drettakis G., Bonneel N., Dachsbacher C., Lefebvre S., Schwarz M., Viaud-Delmon I.: An interactive perceptual rendering pipeline using contrast and spatial masking. In Rendering Techniques 2007 (Proceedings of EGSR) (Grenoble, France, 2007), pp. $297-$ 308.

[DCSD02] Deussen O., Colditz C., Stamminger M., Drettakis G.: Interactive visualization of complex plant ecosystems. In VIS '02: Proceedings of the Conference on Visualization '02 (Boston, MA, USA, 2002), pp. 219 226.

[DDSD03] Décoret X., Durand F., Sillion F. X., Dorsey J.: Billboard clouds for extreme model simplification. $A C M$ Transactions on Graphics (Proceedings of SIGGRAPH 2003), 22, 3 (2003), 689-696.

[DN04] Decaudin P., Neyret F.: Rendering forest scenes in real-time. In Rendering Techniques '04 (Proceedings of EGSR) (Norrköping, Sweden, 2004), pp. 93-102.

[DN09] Decaudin P., Neyret F.: Volumetric billboards. Computer Graphics Forum 28, 8 (2009), 2079-2089.

[DVS03] Dachsbacher C., Vogelgsang C., Stamminger M.: Sequential point trees. ACM Transactions on Graphics (Proceedings of SIGGRAPH 2003) 22, 3 (2003), 657662.

[GCRR11] Gumbau J., Chover M., Remolar I., Rebollo C.: View-dependent pruning for real-time rendering of trees. Computers \& Graphics 35, 2 (2011), 364-374.

[GSSK05] Garcia I., Sbert M., Szirmay-Kalos L.: Leaf cluster impostors for tree rendering with parallax. In Proceedings of the EG Short Presentations (Dublin, Ireland, 2005), pp. 69-72.

[KKF*04] Klein J., Krokowski J., Fischer M., Wand M., Wanka R., Heide F. M. A. D.: The randomized sample tree: a data structure for interactive walk-throughs in externally stored virtual environments. Presence: Teleoper: Virtual Environ. 13, 6 (2004), 617-637.

[LBD07] Luft T., Balzer M., Deussen O.: Expressive illumination of foliage based on implicit surfaces. In Proceedings of the Eurographics Workshop on Natural Phenomena (Prague, Czech Republic, 2007), pp. 71-81.

[LeSt06] Lacewell J. D., Edwards D., Shirley P., Thompson W. B.: Stochastic billboard clouds for interactive foliage rendering. Journal of Graphics, GPU, and Game Tools 11, 1 (2006), 1-12.

[LWC*02] Luebke D., Watson B., Cohen J. D., Reddy M., VARShNey A.: Level of Detail for 3D Graphics. Elsevier Science (New York, NY, USA, 2002).

[Rij79] Russbergen C. J. v.: Information Retrieval (2nd edition). Butterworths, London, 1979.

[RL00] Rusinkiewicz S., Levoy M.: QSplat: A multiresolution point rendering system for large meshes. In Proceedings of the SIGGRAPH 'OO (New York, NY, USA, 2000), pp. 343-352.

[RRCR06] Rebollo C., Remolar I., Chover M., Ripollés O.: An efficient continuous level of detail model for foliage. In Proceedings of 14th International Conference in Central Europe on Computer Graphics, Visualization and Computer Vision (Plzen, Czech Republic, 2006), pp. 335-342.

[SD01] Stamminger M., Drettakis G.: Interactive sampling and rendering for complex and procedural geometry. In Rendering Techniques 2001 (Proceedings of Eurographics Workshop on Rendering (London, UK, 2001), pp. 151-162.

[SSK96] Schaufler G., Stürzlinger W., Kepler J.: A three dimensional image cache for virtual reality. Computer Graphics Forum 15, 3 (1996), 227-236.

[SW08] Scherzer D., Wimmer M.: Frame sequential interpolation for discrete level-of-detail rendering. Computer Graphics Forum (Proceedings EGSR 2008) 27, 4 (June 2008), 1175-1181.

[WFP*01] Wand M., Fischer M., Peter I., Meyer auf der Heide F., Strasser W.: The randomized z-buffer algorithm: interactive rendering of highly complex scenes. In Proceedings of the SIGGRAPH' 01 (New York, NY, USA, 2001), pp. 361-370.

[WS02] Wand M., Strasser W.: Multi-resolution rendering of complex animated scenes. Computer Graphics Forum (Proceedings of Eurographics 2002) 21, 3 (2002), 483491.

[ZP08] Zhang Q.-L., Pang M.-Y.: A survey of modeling and rendering trees. In Edutainment 2008, Z. Pan, X. Zhang, A. E. Rhalibi, W. Woo and Y. Li (Eds.). (Springer Link, Heidelberg, 2008), pp. 757-764. 